\title{
Location of Hubs in a Competitive Environment ${ }^{1}$
}

\author{
VLADIMIR MARIANOV \\ Dept. of Electrical Engineering \\ Universidad Catlica de Chile \\ DANIEL SERRA ${ }^{2}$ \\ Dept. of Economics and Business \\ Universitat Pompeu Fabra \\ Trias Fargas, 25-27, Barcelona 08005, Spain \\ Tel: 34-3-5421666, Fax: 34-3-5421746, E-mail: serra@upf.es \\ Charles ReVelle \\ Dept. of Geography and Environmental Engineering \\ The Johns Hopkins University
}

\begin{abstract}
We offer a formulation that locates hubs on a network in a competitive environment; that is, customer capture is sought, which happens whenever the location of a new hub results in a reduction of the current cost (time, distance) needed by the traffic that goes from the specified origin to the specified destination. The formulation presented here reduces the number of variables and constraints as compared to existing covering models. This model is suited for both air passenger and cargo transportation. In this model, each origindestination flow can go through either one or two hubs, and each demand point can be assigned to more than a hub, depending on the different destinations of its traffic. Links ("spokes" have no capacity limit. Computational experience is provided.
\end{abstract}

keywords: location, hub, tabu search.

\footnotetext{
${ }^{1}$ This research has been partially financed by DCYGIT grant PB95-0980, Ministry of Education (Spain)

${ }^{2}$ Corresponding author
} 


\section{Introduction}

In many transportation or telecommunications networks, the cost of carrying a unit of traffic between two points, decreases as the capacity of the link joining the two points increases. Because of this fact, it is often convenient to design networks in which traffic is concentrated on high capacity links, even if this traffic must travel longer distances. In order to concentrate traffic, each point $i$ offering traffic is connected to a transshipment or switching point through a link. This link carries all the traffic originating at the point $i$, no matter what its destination is, as well as all the traffic whose destination is point $i$, no matter what its origin is. Thus, a first level of concentration takes effect. The transhipment points, called hubs, are in turn interconnected by high-capacity links. Airline passenger flow and cargo delivery networks are examples of networks utilizing hubs. Hubs can be also found in local area computer networks. In large telecommunications networks, switches, concentrators and multiplexers, hubs are found as well.

In spite of its importance, the location of hubs on a network has received a limited attention in the literature (Campbell, 1995; Aykin, 1995). As opposed to other location problems, in this problem an interaction occurs between the facilities to be located. The interaction may make the formulation non-linear (see, for instance, O’Kelly, 1986a; O’Kelly, 1987 or Aykin, 1988) . Alternatively, the interaction squares the number of integer variables, when non-linear products in the objective are replaced by a large number of new variables, (see O'Kelly and Lao, 1991). Simpler versions of the problem are often considered, such as the star-star concentrator location problem. This model assumes a particular class of networks, namely the star-star topology network (Gavish, 1991; Pirkul and Nagarajan, 1992; Pirkul, Narasimhan, De, 1988; Marianov et al, 1995) ,. In this type of network, the hubs are not 
connected to each other, but instead, they are connected to a central point. Thus, there is no interaction between them, and the problem becomes simpler.

Several different forms of hub location problems have been studied in the literature. Planar hub location problems (where hubs can be located anywhere on the plane, but demand is concentrated at discrete points) have been studied by O'Kelly, 1986a; Aykin, 1988; Aykin, 1995. The p-hub-median problem on a network (O’Kelly, 1986b; Klincewicz, 1991) locates a pre-specified number $p$ of hubs on nodes of a network, and allocates the demand points to them. The uncapacitated hub location problem seeks the location of hubs at nodes of a network, and the allocation of demand points to them, so that the sum of investment and operation costs are minimized (O'Kelly, 1992). For reviews of these, as well as other integer programming formulations of discrete hub location problems, see Campbell (1995). Most of the models seek cost minimization. An exception are Campbell's (1995) maximal covering models, that seek the location of $\mathrm{p}$ hubs, so as to maximize the coverage (by pairs of hubs) of weighted demand (represented by origin-destination pairs).

In this paper, we offer a formulation that locates hubs on a network in a competitive environment; i.e. customer capture is sought, which happens whenever the location of a new hub results in a reduction of the time or distance needed by the traffic generated by the traveller to go from origin to destination. The formulation presented here, also reduces the number of variables and constraints as compared to published models. For example, Campbell's 1995 maximal covering model uses $2 n^{2} m^{2}+n^{2}+1$ constraints and $n^{2}+m^{2}+m$ variables, where $n$ is the number of demand points, and $m$ the number of potential locations. In both, the same number of variables need to be declared integer.

This model is better suited for air passenger and cargo transportation, 
among others, in the following situations: 1) an airline has chosen certain airports as hubs, sometime ago. The passenger (or cargo) traffic matrix has changed since the time the hubs were chosen. The airline's management wishes to study the possible relocation of some hubs, given that there are also some competitors. This model can be used considering the existing hubs of the airline, plus the competitor hubs, as competition. New locations can be found that improve current operating costs. b) A small airline wishes to capture some passenger traffic from large companies, by reasoning that large companies optimize their total costs using traffic figures that are very large (corresponding to a very high market share). Thus, there must be some percentage of customers that are not well served (their travel time is longer thatn it could be or, said differently, their optimum is not the optimum of the majority). The model we present is well adapted to these situations.

Another field of applications is express mail services where delivery time is most important. Firms that achieve a lower delivery time than others offer a better service and therefore "capture" more customers. 


\section{The Hub Location Competitive Model (HuLC)}

In the model we present, each origin-destination volume is assigned to exactly one path containing one or two hubs, through which it flows. This assignment results in the allocation of each demand point to possibly more than one hub, although an allocation of each demand point to possibly more than one hub, although an allocation of each demand to a unique hub is possible, by analysis after a solution is found. If this single-hub allocation is forced, the costs obviously increase as compared to the multiple-hub assignment situation. Allocation of demand points to more than one hub have been addressed by O'Kelly and Lao, 1991 and Hall, 1995. We assume that traffic originating at demand point $i$ and having as destination point $j$, can be routed from $i$ to $j$ either through a single hub $k$, or through two hubs $k$ and $l$. We do not allow paths with three or more hubs, as did Fotheringham and O'Kelly, 1989. Links ("spokes") have no capacity limit. The cost per unit of distance of carrying a unit of traffic on an interhub link is $\alpha$ times the cost of carrying the same unit on a demand-hub link, or on a link joining two demand nodes, where $\alpha$ is smaller than one. The $\alpha$ value is used to account for economies of scale. The $0-1$ linear formulation is:

$$
\max Z=\sum_{i \in I} \sum_{j \in J} a_{i j} y_{i j}
$$

Subject to: 


$$
\begin{array}{rlrl}
w_{k l} & \leq x_{k} & k \in K, l \in K \\
w_{k l} \leq x_{l} & k \in K, \quad l \in K \\
y_{i j} \leq \sum_{(k, l) \in N_{i j}} w_{k l} & i \in I, j \in J \\
\sum_{k \in K} x_{k} & =p & & \\
y_{i j} & \in\{0,1\} & i \in I, \quad j \in J \\
x_{k}, w_{k l} & \in\{0,1\} & k \in K, \quad l \in K
\end{array}
$$

where:

$$
\begin{aligned}
i, I= & \text { index and set of origin nodes } \\
j, J= & \text { index and set of destination nodes } \\
k, K= & \text { index and set of candidate nodes } \\
a_{i j}= & \text { traffic, or flow, from } i \text { to } j \\
c_{i j}= & \text { cost of carrying a unit of traffic from node } i \text { to node } j \\
C_{i j}= & \text { current competitor's cost of carrying a unit of traffic from node } i \text { to node } j \\
& \text { where the } C_{i} j \text { applies to the lowest cost of all competing airlines } \\
\alpha= & \text { cost reduction factor for flows between hubs }
\end{aligned}
$$

$$
\begin{aligned}
& N_{i j}=\left\{(k, l) \| c_{i k}+\alpha c_{k l}+c_{l j} \leq C_{i j}\right\} \\
& \text { that is, } N_{i j} \text { is the set of pair of points } k, l \text { such that, } \\
& \text { if hubs were located at them, capture of traffic between } \\
& i \text { and } j \text { result, because the cost of carrying that traffic } \\
& \text { would increase as compared with the current cost; } \\
& p=\text { total number of available hubs } \\
& x_{k}=0,1 ; 1 \text {, if there is a hub in node } k ; 0 \text {, otherwise } \\
& w_{k l}=0,1 ; 1 \text {, if there is a hub in nodes } k \text { and } l ; 0 \text {, otherwise } \\
& y_{i j}=0,1 ; 1 \text {, if the flow from } i \text { to } j \text { is captured; } 0 \text {, otherwise }
\end{aligned}
$$

The objective (1) maximizes the sum of captured flows. Constraints (2) 
and (3) define the variable $w_{k l}$, which is zero unless hubs are located at $k$ and $l$. Constraint (4) allows demand or traffic between nodes $i$ and $j$ to be captured, only if hubs are located at $k$ and $l$ where $k$ and $l$ are in the "capture set" $N_{i j}$. By "capture set" we mean the pair of potential locations $(k, l)$ such that the cost of going from $i$ to $j$ through at least one of them is less than the current cost of going through competitor's system. In order to allow the flow to go through only one hub, the pair $(k, k)$ is also included in the set, with the variable $w_{k k}$ being allowed as well. Finally, constraint (5) sets the number of hubs to be located.

The HuLC model fixes the number of hubs to be located. If the number of hubs is not pre-specified, and if there is information about annual amortized opening and continuing costs $f_{j}$ for each potential hub location $j$, constraint (5) can be dropped, and a new objective can be structured as follows:

$$
\max Z=\sum_{i \in I} \sum_{\in J} r_{i j} y_{i j}-\sum_{k \in K} f_{k} x_{k}
$$

where $r_{i j}$ is the expected annual revenue from flow $(i, j)$. This objective could stand along side the capture objective or replace it.

As was already mentioned, this model can be used for the relocation and/or addition of hubs. In order to do so, it can be slightly modified. Let $p_{o}$ be the current number of hubs, and $K_{0}$ the index set of their locations. Let $p_{s}$ be the number of hubs that are to stay at their current location, $p_{r}$ the number of hubs to be relocated and $p_{n}$ the number of totally new hubs. Thus, $p_{o}=p_{s}+p_{r}$. Then, constraint (5) is replaced by the following two constraints:

$$
\begin{aligned}
\sum_{k \in K} x_{k} & =p_{s}+p_{r}+p_{n} \\
\sum_{k \in K_{o}} x_{k} & =p_{s}
\end{aligned}
$$

The first of these constraints states that the total final number of hubs will 
be $p_{0}+p_{n}$. The second one forces $p_{s}$ hubs to stay at their current positions.

A further modification is possible. In the previous model, a flow is either captured or not captured, depending on the new cost of carrying it as compared to the competitor's cost of carrying the same flow from its origin to its destination. It is possible to define capture in a less granular form. For example, three levels of capture might be defined as follows:

- Level 1: if $0.9 C_{i j}<c_{i k}+\alpha c_{k l}+c_{l j} \leq 1.1 C_{i j} j$ then a $50 \%$ of the flow is captured;

- Level 2: if $0.7 C_{i j}<c_{i k}+\alpha c_{k l}+c_{l j} \leq 0.9 C_{i} j$ then a $75 \%$ of the flow is captured;

- Level 3: if $c_{i k}+\alpha c_{k l}+c_{l j} \leq 0.7 C_{i j}$ then a $100 \%$ of the flow is captured;

Using the same principle, more than three levels can be defined. For each level, a capture set and a capture variable is defined. In the example, the following sets are defined:

$$
\begin{aligned}
N_{i j}^{50} & =\left\{k, l \mid 0.9 C_{i j}<c_{i k}+\alpha c_{k l}+c_{l j} \leq 1.1 C_{i j}\right\} \\
N_{i j}^{75} & =\left\{k, l \mid 0.7 C_{i j}<c_{i k}+\alpha c_{k l}+c_{l j} \leq 0.9 C_{i j}\right\} \\
N_{i j}^{100} & =\left\{k, l \mid c_{i k}+\alpha c_{k l}+c_{l j} 0.7 C_{i j}\right\}
\end{aligned}
$$

together with the variables

$$
\begin{aligned}
y^{50} & =1, \text { if the flow from } i \text { to } j \text { is } 50 \% \text { captured, } 0 \text { otherwise } \\
y^{75} & =1 \text {, if the flow from } i \text { to } j \text { is } 75 \% \text { captured, } 0 \text { otherwise } \\
y^{100} & =1 \text {, if the flow from } i \text { to } j \text { is } 100 \% \text { captured, } 0 \text { otherwise }
\end{aligned}
$$

The model takes the form: 


$$
\max Z=\sum_{i \in I} \sum_{j \in J} a_{i j} y_{i j}^{100}+0.75 a_{i j} y_{i j}^{75}+0.50 a_{i j} y_{i j}^{50}
$$

Subject to:

$$
\begin{array}{rlrl}
w_{k l} & \leq x_{k} & k \in K, l \in K \\
w_{k l} \leq x_{l} & k \in K, \quad l \in K \\
y_{i j}^{100} \leq \sum_{(k, l) \in N_{i j}^{100}} w_{k l} & i \in I, j \in J \\
y_{i j}^{75} \leq \sum_{(k, l) \in N_{i j}^{75}} w_{k l} & i \in I, j \in J \\
y_{i j}^{50} \leq \sum_{(k, l) \in N_{i j}^{50}} w_{k l} & i \in I, j \in J \\
y_{i j}^{100}+y_{i j}^{75}+y_{i j}^{50} & \leq 1 \\
\sum_{k \in K} x_{k} & =p & \\
y_{i j} & \in\{0,1\} \\
x_{k}, w_{k l} & \in\{0,1\} & \\
\{i \in I, \forall j \in J & \\
&
\end{array}
$$

In this formulation, the objective reflects the different levels of capture, while constraints (7), (8) and (9) define each level. Constraint (10) forces each flow to be captured at one level only.

The previous HuLC formulation presented envolves a large number of variables and constraints. For a network of 20 nodes, where all nodes are demand nodes and possible hub locations, and adding up traffic in both directions between each pair of nodes, there are 820 variables and 801 constraints. On the other hand, as it will be shown in the section corresponding to computational 
experience, the problem is not integer friendly. In the next section we propose a meta-heuristic to solve this combinatorial problem.

\section{A Tabu Heuristic to Solve the Model}

Several heuristics for the solution of the hub problem have been studied by O’Kelly (1987), Skorin-Kapov and Skorin-Kapov (1994), Aykin (1994) and Klincewicz, $(1989,1991)$. In order to solve the HULC problem a metaheuristic is presented, based on the well-known Teitz and Bart (1968) one-opt heuristic, improved by Densham and Rushton (1992), and modified with some Tabu search.

The flow process has three phases. In the first phase, an initial solution is obtained using a greedy adding heuristic where at each iteration a hub is located in the node than gives the best marginal improvement in the objective without violating the constraint set. Phase one is over when $p$ hubs are located. Then, in the second phase, a Teitz and Bart heuristic is used. At each iteration a hub is moved from its current position to another potential hub location. The objective is computed and the new set of positions is kept as the current solution if the move has improved the objective. If the objective is not improved, the solution before the one-opt trade is restored. If at the end of a complete cycle of all trades the objective has not improved, the heuristic is over. Otherwise, the process is restarted. Of course, the solution obtained with this heuristic may not be optimal. In the third phase a tabu process is used, with its initial solution the one found in the second phase. Essentially, the tabu heuristic explores a piece of the solution space through a repeated examination of all solution neighbors. The search my move to a neighboring solution even if the objective value at this neighbor has a worse value of the objective (Glover 1977,1989, 1990). This approach 
aids in avoiding being trapped in a local optimum. In order to avoid cycling solutions that have recently been examined, solutions are inserted in a tabu list that is constantly updated.

This method has been successfully applied to a wide variety of optimization problems (see, for example, Skorin-Kapov (1990), Gendreau et al. (1994) and Glover and Laguna (1993).

A more detailed description of the algorithm follows. Let $W_{t}$ be the set of locations $w_{j}, w_{j}>0$.

\section{Phase 1}

1. Set $W_{0}:=\emptyset$ and $p:=1$.

2. Set $W_{p}:=W_{p-1} \cup v_{k}$, where $v_{k}$ represents the index of the node that gives the largest increase in flow capture:

$$
\max _{v_{k} \in V}\left[z\left(W_{p-1} \cup v_{k}\right)-z\left(W_{p-1}\right)\right]
$$

Set $p:=p+1$ a repeat step 2. Stop when $p=\bar{p}$

\section{Phase 2}

1. Set $W^{*}:=W_{p}$ and $z_{W}^{*}:=z\left(W_{p}\right)$

2. Set $t:=0, z_{W}^{0}=z_{W}^{*}$.

3. Set $W_{t}:=W_{t-1}-v_{k}+v_{l}$, where $v_{k} \in W_{p}$ and $v_{l} \in\left(V-W_{t-1}\right)$.

4. If $z\left(W_{t}\right)>z_{W}^{*}, \quad W^{*}:=W_{t}$ and $z_{W}^{*}:=z\left(W_{t}\right)$, repeat step 2 until all nodes and hubs have been exchanged.

5. If $z_{W}^{*}>z\left(W_{0}\right)$, set $z_{W}^{0}:=z_{W}^{*}$ and repeat step 2. Otherwise, go to phase 3 . 


\section{Phase 3}

1. Set again $t:=0$.

2. Set $z_{W}^{0}:=z_{W}^{*}$. No node is tabu.

3. Consider all solutions of adjacent nodes $W_{t}^{i}$ of $W_{t}$, obtained by exchanging a hub from node $v_{i}^{\prime} \in W_{t}$ to node $v_{i}^{\prime \prime} \notin W_{t}$. Relabel the solutions $W_{t}^{i}$ in decreasing order of $z\left(W_{t}^{i}\right)$. Relabel all vertices accordingly. Set $i:=i+1$.

4. If $z\left(Y_{t}^{i}\right)>z_{W}^{*}$ or if $v_{i}^{\prime \prime}$ is not tabu, set $W_{t+1}=W_{t}, z_{W}^{*}:=z\left(W_{t}\right)$, declare $v_{i}^{\prime}$ tabu until $t+\theta$, where $\theta$ is a pre-fixed value, and go to step 5. Otherwise, set $i:=i+1$. If $i$ is larger than the number of adjacent solutions, set $i$ equal to the index of the vertex $v_{i}^{\prime \prime}$ with the lowest tabu $\operatorname{tag} t+\theta$ and lift the tabu status of $v_{i}^{\prime \prime}$. Repeat step 3 .

5. Set $t:=t+1$. If $t$ is less than a pre-fixed upper bound $T$, go to step 2 of phase 3. Otherwise, set $p:=p+1$ and go to step 2 of phase 1 if the solution found is feasible. If in the last iteration no feasible solution is found, stop.

In step 2 of phase 3 , the tabu status can be canceled if this implies an improvement in the objective. This rule is known as aspiration criterion.

In the following section some computational experience is presented.

\section{Computational Experience}

All numerical tests were carried on a PC-pentium 75 with a $24 \mathrm{Mb} \mathrm{RAM}$ memory. The algorithm was coded in FORTRAN. We used both LINDO and MINOS 5.1 for solving problems. The computational comparisons are based 
randomly generated networks as well as the AP (Australian Post) data set (Ernst and Krishnamoorthy 1996).

First, 20, 2540 and 50 networks from the 200-node AP data set (which is available at http://mscmga.ms.ic.ac.uk) were generated following Ernst and Krishnamoorthy's method. The locations of the competitor hubs were the optimal solutions obtained by Ernst and Krishnamoorthy for the uncapacitated multiple-allocation p-hub median problem (Ernst and Krishnamoorthy, forthcoming).

We first tried to solve the model using linear programming relaxation and branch and bound, but with little success even for small networks. For a 10 node network the model took on average 20 minutes to obtain the optimal solution, and for a 20 node network the execution was aborted without reaching an optimum after 8 hours. We also tried to solve the model by replacing constraints (2) and (3) by the following:

$$
\begin{array}{ll}
\sum_{l \in K} w_{k l} \leq|K| x_{k} & \forall k \in K \\
\sum_{k \in K} w_{k l} \leq|K| x_{l} & \forall l \in K
\end{array}
$$

where $|K|$ is the cardinality of $K$, that is, the number of elements in $K$, but no improvement in the computing times were obtained. In this sense, this model belongs to the INP class (Integer Nasty Programming). We used complete enumeration on these small problems to obtain optimal solutions and compared them to the solutions obtained by the heuristic. Results are presented in Table 1. 
Table 1: Results, AP data

\begin{tabular}{|cc|cc|ccc|cc|c|}
\hline$n$ & $p, q$ & Loc. A & Loc. B & OBJ. A & OBJ. B & $\%$ Share & Opt. & Imprv. & Time \\
\hline 20 & 2 & 14,15 & 14,16 & 1543 & 2436 & $39 \%$ & yes & no & $2 \mathrm{~s}$ \\
& 3 & $7,14,15$ & $6,12,14$ & 1718 & 2261 & $43 \%$ & yes & no & $5 \mathrm{~s}$ \\
& 4 & $7,13,14,15$ & $2,6,12,14$ & 2079 & 1850 & $52 \%$ & yes & no & $11 \mathrm{~s}$ \\
& 5 & $7,9,14,15,19$ & $2,6,12,13,14$ & 2000 & 1978 & $50 \%$ & yes & no & $22 \mathrm{~s}$ \\
\hline 25 & 2 & 7,19 & 8,18 & 1486 & 2493 & $37 \%$ & yes & no & $3.24 \mathrm{~s}$ \\
& 3 & $17,18,19$ & $2,8,18$ & 1981 & 1998 & $50 \%$ & yes & no & $9.28 \mathrm{~s}$ \\
& 4 & $7,16,18,19$ & $2,8,17,18$ & 1765 & 2214 & $44 \%$ & yes & no & $19.32 \mathrm{~s}$ \\
& 5 & $2,8,14,17,19$ & $2,8,17,18,20$ & 2770 & 1209 & $70 \%$ & yes & yes & $37 \mathrm{~s}$ \\
\hline 40 & 2 & 28,29 & 12,18 & 1617 & 2362 & $41 \%$ & yes & no & $7.46 \mathrm{~s}$ \\
& 3 & $27,28,29$ & $12,23,28$ & 1757 & 2222 & $44 \%$ & yes & no & $23 \mathrm{~s}$ \\
& 4 & $12,17,28,29$ & $12,23,26,28$ & 1728 & 2251 & $43 \%$ & yes & yes & $53 \mathrm{~s}$ \\
\hline 50 & 2 & 15,36 & 14,35 & 1654 & 2325 & $41 \%$ & yes & no & $13.24 \mathrm{~s}$ \\
& 3 & $6,32,36$ & $14,28,35$ & 1615 & 2364 & $40 \%$ & yes & yes & $41.58 \mathrm{~s}$ \\
& 4 & $6,33,35,36$ & $14,28,32,35$ & 1633 & 2346 & $41 \%$ & yes & yes & $89 \mathrm{~s}$ \\
\hline
\end{tabular}

In the first and second columns the number of nodes and the number of hubs for each firm are presented respectively. $\mathrm{A}$ is the entering firm and $\mathrm{B}$ is the firm in place. The final locations for the entering firm obtained by the heuristic are shown in the third column. In the fourth column the location of the competitor's hub are also presented. The final flow capture for both firms are presented in absolute terms in columns 4 and 5 , and in the sixth column the percentage capture by Firm $\mathrm{A}$ is shown. The seventh column indicates if the solution of the heuristic is optimal. The eighth column indicates if the third phase of the heuristic improved the solution obtained so far by the second phase. Finally, computer times for the heuristic are shown in the last column.

The heuristic obtained optimal solutions in all the test runs. $90 \%$ of these solutions were obtained by phase II. In general, the flow capture by the entering firm was below $50 \%$, even though the same number of hubs as the competitor firm was used. This is due most probably to the good positioning 
of the competitor hubs and to the fact that, in case of ties between both firms, the flows were fully assigned to the original competitor firm.

An example of flow capture is presented in figure 1 for $n=20$ and $p, q=3$. Competitor hubs are located in nodes 6, 12 and 14 and the entering hubs are located in nodes 7, 14 and 15. The arrows denote the assignments for departures in the top picture and arrivals in the bottom picture.

\section{Conclusions}

In this paper a new Hub location model has been formulated. This model locates hubs so as to maximize the flow capture when there are competitors already operating in the market. A heuristic to solve the problem has been proposed and tested in diferent networks. Its performance in the test runs is quite satisfying.

The model assumes that, if the cost $c_{i j}$ for the entering firm of going from $i$ to $j$ is lower than any of the competitors $\left(C_{i j}\right)$, then its flow is fully captured. This assumption can be modified to assume that the flow capture is a function of the difference between the cost of the entering firm and the competitor's cost. For example, it can be assumed that if $c_{i j}=C_{i j}$, then the flow is equally divided among both firms, and if $c_{i j}$ is $x \%$ lower than $C_{i j}$, then the flow capture of the entering firm is equal to $50 \%+x \%$ of the flow from $i$ to $j$. While in the mathematical formulation of the problem this would lead to a non-linear problem, it can be easily incorporated in the heuristic when the capture objective is computed in each iteration.

it is also shown how it is possible to change this assumption, allowing different percentages of capture, depending on the relation between costs of using the entering firm or the competitor. So, there can exist several different levels of capture. This situation can be also easily incorporated in the heuristic 
when the capture objective is computed in each iteration. The model can also be adapted to relocate or add hubs to an existing network. 


\section{References}

[1] T. Aykin. On the location of hub facilities. Transportation Science, $22: 155-157,1988$.

[2] T. Aykin. Lagrangian relaxation based approaches to capacitated huband-spoke network design problems. European Journal of Operational Research, 79:501-523, 1994.

[3] T. Aykin. The hub location and routing problem. European Journal of Operational Research, 83:200-219, 1995.

[4] J.F. Campbell. Integer programming formulations of discrete hub location problems. European Journal of Operational Research, 72:387-405, 1995.

[5] P. Desham and G. Rushton. Strategies for solving large locationallocation problems by heuristic methods. Environment and Planning A, 24:289-304, 1992 .

[6] A.T. Ernst and M. Krishamoorthy. Efficient algorithms for the uncapacitated multiple allocation $p$-hub median problem. European Journal of Operational Research, forthcoming, 1997.

[7] A.T. Ernst and M. Krishamoorthy. Efficient algorithms for the uncapacitated single allocation $p$-hub median problem. Location Science, 4(3):139-154, 1997.

[8] A.S. Fotheringham and M.E: O'Kelly. Spatial interaction models: Formulation and applications. Kluwer Academic Publishers, Boston, M.A., 1989. 
[9] B. Gavish. Topological design of telecommunications networks - local access design methods. Annals of Operations Research, 33:17-71, 1991.

[10] M. Gendreau, A. Hertz, and G. Laporte. A tabu search algorithm for the vehicle routing problem. Management Science, 40:1276-1290, 1994.

[11] F. Glover. Heuristic for integer programming using surrogate constraints. Decision Sciences, 8:156-166, 1977.

[12] F. Glover. Tabu search, part i. ORSA Journal of Computing, 1:190-206, 1989.

[13] F. Glover. Tabu search, part ii. ORSA Journal of Computing, 2:4-32, 1990.

[14] F. Glover and M. Laguna. Tabu search. In C. Reeves, editor, Modern heuristics techniques for combinatorial problems. Blackwell, Oxford, 1993.

[15] R.W: Hall. Configuration of an overnight air package network. Transportation Research, 23:139-149, 1995.

[16] J.G. Klincewicz. Avoiding local optima in the p-hub location problem using tabu search and grasp. ATEST Bell Laboratories, 1989.

[17] J.G. Klincewicz. Heuristics for the p-hub location problem. European Journal of Operational Research, 53:25-37, 1991.

[18] V. Marianov, M. Perez, and M. Rios. Location of nodes on communications networks considering a user pre-specified minimum reliability under failures. In Third International Conference on Telecommunications Systems: Modelling and Analysis, Nashville, Ten., 1995. 
[19] M.E. O'Kelly. Activity levels at hub facilities in interacting networks. Geographical Analysis, 18:343-356, 1986.

[20] M.E. O'Kelly. The location of interacting hub facilities. Transportation Science, 20:92-106, 1986.

[21] M.E. O'Kelly. A quadratic integer program for the location of interacting hub facilities. European Journal of Operational Research, 32:393-404, 1987.

[22] M.E. O'Kelly. Hub facility location with fixed costs. Papers in Regional Science: The Journal of the RSAI, 71:293-306, 1992.

[23] M.E. O'Kelly and Y. Lao. Mode choice in a hub-and-spoke network: A zero-one linear programming approach. Geographical Analysis, 23(4):283-297, 1991.

[24] H. Pirkul and V. Nagarajan. Locating concentrators in centralized computer networks. Annals of Operations Research, 36:247-262, 1992.

[25] H. Pirkul, S. Narasimhan, and P. De. Locating concentrators for primary and secondary coverage in a computer communications network. IEEE Transactions on Communications, 36(4):247-262, 1988.

[26] D. Skorin-Kapov and J. Skorin-Kapov. On tabu search for the location of interacting hub facilities. European Journal of Operational Research, 73:502-509, 1994.

[27] J. Skorin-Kapov. Tabu search applied to the quadratic assignment problem. ORSA Journal on Computing, 2:33-45, 1990. 
[28] M. B. Tietz and P. Bart. Heuristic methods for estimating the generalized vertex median of a weighted graph. Operations Research, 16:955-965, 1968. 
Figure 1: Example with $n=20$ and $p, q=3$

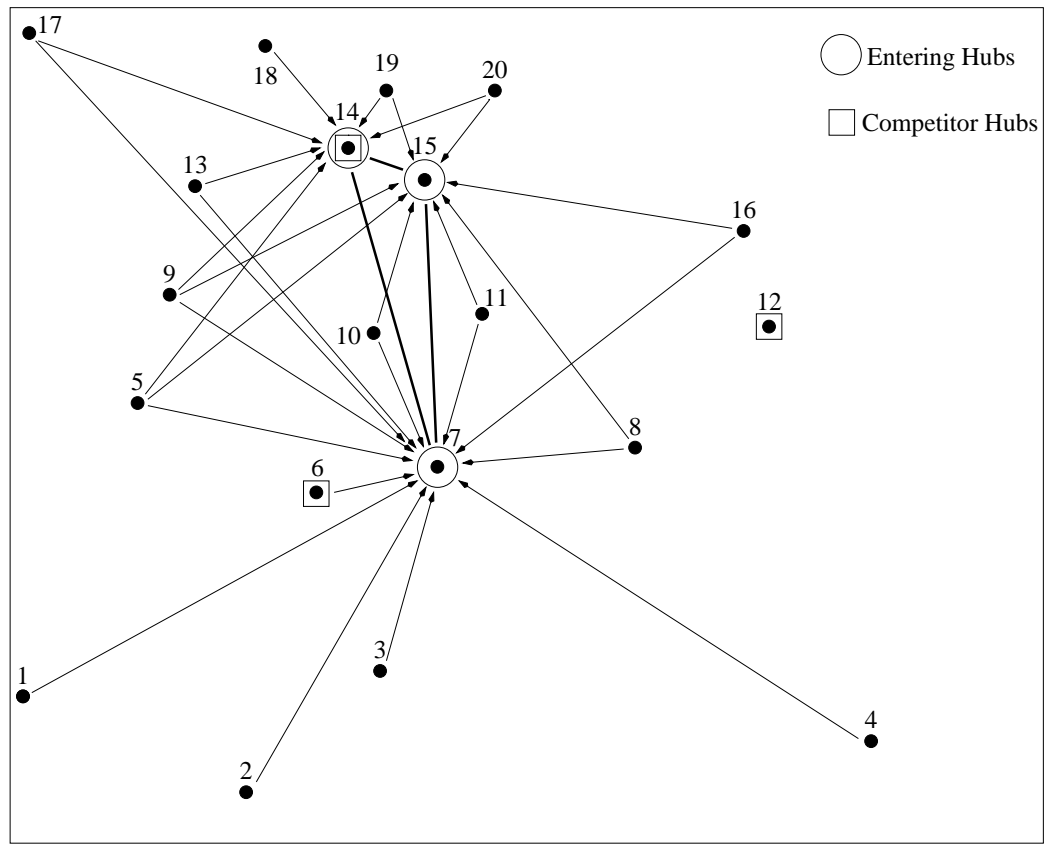

DEPARTURES

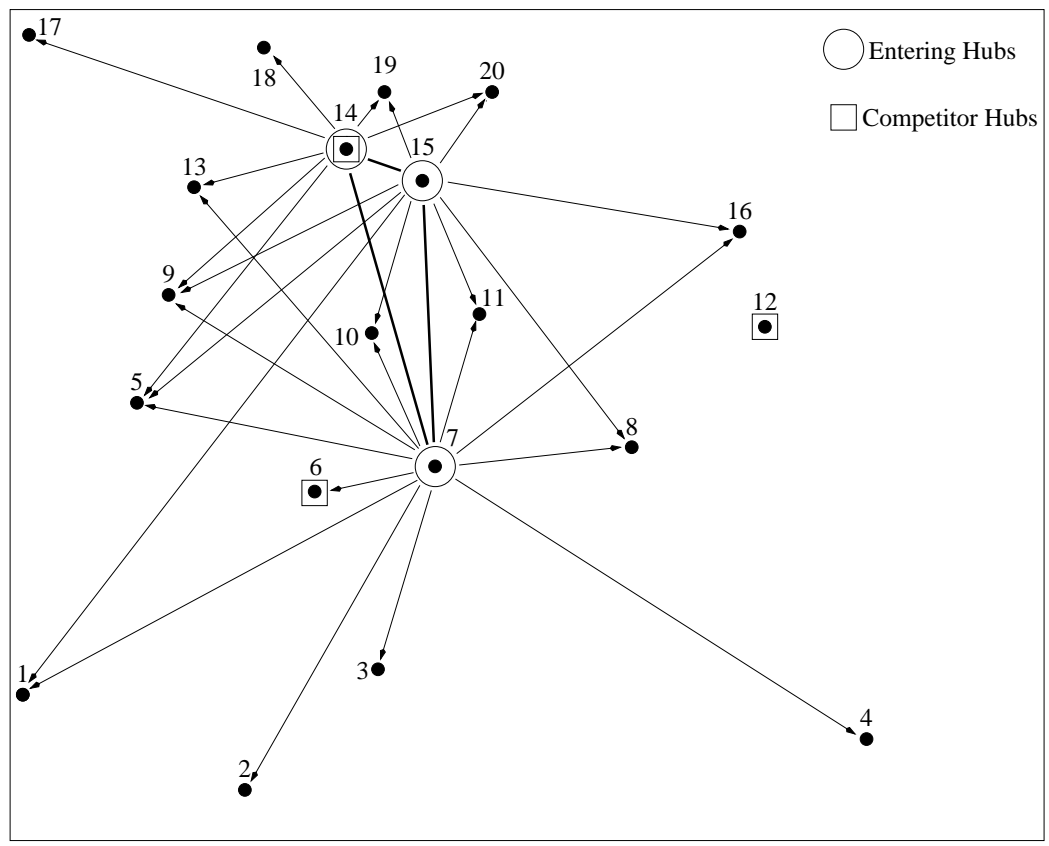

ARRIVALS 